\title{
NOTE ON LOSSES IN SHEET IRON AT RADIO FREQUENCIES*
}

\author{
$\mathrm{Br}$ \\ Marius. Latour \\ (Paris, France)
}

I. The study of Foucault currents in iron sheets at high frequency was made first by Oliver Heaviside, and later by J. J. Thomson. ${ }^{1}$

Recently, Mr. Bethenod ${ }^{2}$ has considered the complication caused by the phenomenon of hysteresis, and has introduced that phenomenon in his calculations by utilizing the method of procedure first employed by Ferraris (1888), according to which hysteresis is supposed to cause a constant lag $\tau$ of phaseangle between the magnetic induction $\boldsymbol{B}$ and the magnetizing field $\boldsymbol{H}$. In seeking to determine the final phase-lag between the emf. and the current in a coil having a closed magnetic circuit, when the frequency is increased indefinitely, Mr. Bethenod has shown that this limiting phase-lag, instead of being equal to $\frac{\pi}{4}$, as indicated by the formulas of J. J. Thomson, is diminished by an angle equal to $\frac{\tau}{2}$ by the effect of hysteresis. In reality, Mr. Bethenod's conclusion, according to which the limiting angle of phase-lag, between the emf. and the current, is diminished thru hysteresis by an angle equal to $\frac{\tau}{2}$, does not seem to be related to any particular interpretation of the phenomenon of hysteresis. It is natural, in fact, that the losses due to hysteresis, as with the losses due to Foucault currents, should tend to increase the "watt" current absorbed, and, consequently, should tend to bring the current more nearly in phase with the emf.

Before proceeding to any calculation, it is easy to under-

* Received by the Editor, October 15, 1918. (This paper was also delivered before the Societé Internationale des Electriciens.)

1 "The Electrician," volume 28, 1892, page 599.

2 "La Lumière Electrique," July 22, 1916, volume 34, 2nd Series, page 73. 
stand how hysteresis influences Foucault currents, and to understand how Foucault currents may influence hysteresis losses. If we assume that hysteresis introduces a phase-lag between magnetic induction and ampere-turns, we can understand at once that the introduction of Foucault currents into the equations is thereby affected. On the other hand, the presence of Foucault currents causes the magnetic induction in iron sheets to vary from the center to the external surface; and since the losses due to hysteresis increase according to a power of the magnetic induction which is higher than the first power, the losses will be higher than if the magnetic induction in the sheet were assumed to remain uniform. The hysteresis losses, therefore, depend on the influence of Foucault currents on the distribution of magnetic induction.

It will be principally necessary to take into consideration this distribution of magnetic induction in the sheet in order to calculate the losses due to hysteresis. In particular, if we assume these losses to be proportional to the square of the magnetic induction, which is all the more likely because, at high frequency, the magnetic induction is always low, it will be necessary to know, somehow, the effective spatial distribution of the magnetic induction in the sheet.

The purpose of the author is to revise the methods of dealing mathematically with Foucault currents, and to study the influence of hysteresis on losses due to Foucault currents, as well as to study the losses due to hysteresis itself. His purpose also is to obtain formulas which are useful for the study of radio frequency apparatus.

II. To establish the equations for Foucault currents, the author will not introduce directly the general equations of Maxwell, as is usually done; these equations can be established by equivalent considerations which are much more familiar to engineers.

Let us consider (Figure 1) a sheet of thickness 2a. Let us take as origin the median plane $X X$, parallel to the two faces of the sheet, $S$ and $S^{\prime}$. On account of symmetry, the Foucault currents which go thru the sheet will give rise to currents of equal densities, and of opposite polarities at symmetrical points $x$ and $x^{\prime}$, situated at equal distances, $O x$ and $O x^{\prime}$, from the origin $O$. The Foucault currents which tend to form a shield against the magnetic flux which is passing thru the sheet, in a direction parallel to the faces $S$ and $S^{\prime}$, follow the directions indicated 
by arrows in the plane of the figure. It should be noted, moreover, that the ampere-turns acting to produce a magnetic field at the point $x$, are those due to currents circulating in the region at the right of $O x$ and at the left of $O x^{\prime}$. The currents circulating

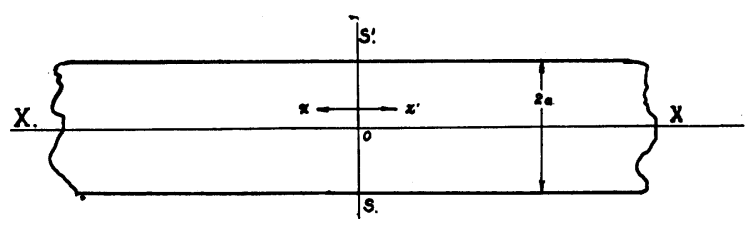

Figure 1

within the space bounded by the planes parallel to $X X$, which pass thru $x$ and $x^{\prime}$, produce, in fact, no field external to that space. Let us designate by $\delta$ the current-density in the sheet at the point $x$. The decrease in ampere-turns per centimeter, which results in passing from a thickness $d x$ toward the external surface $S$ of the sheet, is $\delta d x$. The magnetic induction $\boldsymbol{B}$ at the point $x$ will be decreased by a corresponding amount $d \boldsymbol{B}$ such that

$$
d \boldsymbol{B}=-4 \pi \mu \delta d x
$$

whence

$$
\frac{d \boldsymbol{B}}{d x}=-4 \pi \mu \delta
$$

In reality, the current density $\delta$ and the magnetic induction $B$, being both harmonic functions of time, can be expressed as follows:

$$
\begin{gathered}
\delta=\delta_{1} \sin \omega t-\delta_{2} \cos \omega t \\
\boldsymbol{B}=\boldsymbol{B}_{1} \sin \omega t-\boldsymbol{B}_{2} \cos \omega t
\end{gathered}
$$

in which equations any origin can be taken arbitrarily for time values $(t)$. Under those conditions equation (1) corresponds to the two following equations:

$$
\begin{aligned}
& \frac{d \boldsymbol{B}_{1}}{d x}=-4 \pi \mu \boldsymbol{\delta}_{1} \\
& \frac{d \boldsymbol{B}_{2}}{d x}=-4 \pi \mu \boldsymbol{\delta}_{2}
\end{aligned}
$$

If we assume that hysteresis introduces an angle of phaselag, $\tau$, between magnetic induction and ampere-turns, we can 
say that everything happens, as far as the ampere-turns are concerned, as if the current density, $\delta$, corresponded to a fictitious density, $\boldsymbol{\delta}$, having a phase-lag, $\tau$, such that

$$
\begin{aligned}
\delta^{\prime} & =\grave{o}_{1} \sin (\omega t-\tau)-\grave{o}_{2} \cos (\omega t-\tau) \\
& =\left(\grave{\delta}_{1} \cos \tau-\grave{o}_{2} \sin \tau\right) \sin \omega t-\left(\grave{\partial}_{2} \cos \tau+\delta_{1} \sin \tau\right) \cos \omega t
\end{aligned}
$$

Equations (2) then become

$$
\begin{aligned}
& \frac{d \boldsymbol{B}_{1}}{d x}=-4 \pi \mu\left(\boldsymbol{\delta}_{1} \cos \tau-\grave{\delta}_{2} \sin \tau\right) \\
& \frac{d \boldsymbol{B}_{2}}{d x}=-4 \pi \mu\left(\delta_{2} \cos \tau+\delta_{1} \sin \tau\right)
\end{aligned}
$$

The choice between equations (2) and $\left(2^{\prime}\right)$ will then depend on whether the phenomenon of hysteresis is to be taken into consideration or not, in the equations for Foucault currents.

We now proceed to establish a second equation by a simple consideration which follows.

The emf. induced per centimeter in the direction $X X$ between the two planes which pass thru the abscissa points $x$ and $x+d x$ is

$$
-\frac{d}{d t} \boldsymbol{B} d x=-\omega\left(\boldsymbol{B}_{1} \cos \omega t+\boldsymbol{B}_{2} \sin \omega t\right) d x
$$

This emf. must be exactly balanced by the difference between the ohmic drop per centimeter in the plane passing thru $x$ and the ohmic drop per centimeter in the plane passing thru $x+d x$. This difference is equal to $\rho d \dot{\delta}$, where $\dot{o}$ designates the resistivity of the sheet. We therefore have

$$
\begin{aligned}
&-\omega\left(\boldsymbol{B}_{1} \cos \omega t+\boldsymbol{B}_{2} \sin \omega t\right) d x=\varrho d \boldsymbol{\boldsymbol { \partial }} \\
&=\left(\frac{d}{d \bar{x}} \cdot \sin \omega t-\frac{d \boldsymbol{\delta}_{2}}{d x} \cos \omega t\right) d x
\end{aligned}
$$

From this we obtain the two following equations:

$$
\begin{aligned}
& \omega B_{1}=\rho \frac{d \grave{o}_{2}}{d x} \\
& \omega B_{2}=-\rho \frac{d \partial_{1}}{d x}
\end{aligned}
$$

From equations $\left(2^{\prime}\right)$ and (4) we obtain the two following equations of the second order:

$$
\begin{aligned}
& \frac{d^{2} \grave{\partial}_{1}}{d x^{2}}=\frac{4 \pi \mu \omega}{\rho}\left(\grave{\partial}_{2} \cos \tau+\grave{o}_{1} \sin \tau\right) \\
& \frac{d^{2} \grave{\partial}_{2}}{d x^{2}}=-\frac{4 \pi \mu \omega}{\rho}\left(\grave{\partial}_{1} \cos \tau-\grave{\partial}_{2} \sin \tau\right)
\end{aligned}
$$


Taking

$$
\frac{4 \pi \mu(1)}{\rho}=2 m^{2}, \quad \sqrt{1+\sin \tau}=\alpha, \quad \sqrt{1-\sin \tau}=\beta
$$

we obtain, by integration, the following:

$$
\begin{aligned}
& \delta_{1}=-A \frac{e^{\max }-e^{-\max }}{2} \cos m \beta x=-A \sinh m \alpha x \cos m \beta x \\
& \delta_{2}=A \frac{e^{\max }+e^{-\max }}{2} \sin m \beta x=A \cosh m \alpha x \sin m \beta x
\end{aligned}
$$

in which $A$ is a constant of integration. ${ }^{3}$

From these and from equation (4), the values of $\boldsymbol{B}_{1}$ and $\mathbf{B}_{2}$ are obtained:

$$
\begin{aligned}
& \boldsymbol{B}_{1}=\frac{A \rho m}{\omega}(\alpha \sinh m \alpha x \sin m \beta x+\beta \cosh m \alpha x \cos m \beta x) \\
& \boldsymbol{B}_{2}=-\frac{A \rho m}{\omega}(\beta \sinh m \alpha x \sin m \beta x-\alpha \cosh m \alpha x \cos m \beta x)
\end{aligned}
$$

From the above solution (6) we obtain the maximum current density value $\boldsymbol{\delta}_{\max }$ at the point $x$ :

$$
\delta_{\max }=\sqrt{\delta_{1}^{2}+\delta_{2}^{2}}=\frac{A}{\sqrt{2}} \sqrt{\cosh 2 m \alpha x+\cos 2 m \beta x}
$$

From solution (7) we obtain the maximum value of magnetic induction $\boldsymbol{B}_{\max }$ at the point $x$ :

$$
\boldsymbol{B}_{\max }=\frac{A \rho m}{\omega} \sqrt{\cosh 2 m \alpha x+\cos 2 m \beta x}
$$

We can now determine the constant $A$ by starting either from the apparent, or the mean magnetic induction in the sheet, or from the external ampere-turns per centimeter $(J)$ which act on the sheet.

When starting from the apparent magnetic induction $\boldsymbol{B}_{a p p}$ it is to be noted that the emf. per centimeter, which must equal the ohmic drop due to Foucault currents along the external surface of the sheet, is $\boldsymbol{\omega} \boldsymbol{B}_{a p p} \alpha$. We therefore have:

Whence:

$$
\omega \boldsymbol{B}_{a p p} \alpha=\frac{\rho A}{\sqrt{2}}(\cosh 2 m \alpha a-\cos 2 m \beta a)^{\frac{1}{2}}
$$

$$
A=\frac{\sqrt{2} \bar{\omega} a \boldsymbol{B}_{a p p}}{\varrho(\cosh 2 m \alpha a-\cos 2 m \beta a)^{\frac{1}{2}}}
$$

${ }^{3}$ The general integral would require a second constant, but it is found that this constant must be equal to zero in order that the condition of symmetry implying $\delta_{1}=\delta_{2}=0$ should be satisfied for $x=0$. 
When starting from the external ampere-turns, $J$, per centimeter, it must be noted that the magnetic induction at the surface of the sheet must be equal to $4 \pi \mu J$. We therefore have:

$$
4 \pi \mu J=\frac{A \rho m}{\omega}(\cosh 2 m \alpha a+\cos 2 m \beta a)^{\frac{1}{2}}
$$

Whence:

$$
A=\frac{\omega}{g m} \frac{4 \pi \mu J}{(\cosh 2 m \alpha a+\cos 2 m \beta a)^{\frac{1}{2}}}
$$

Apparent Permeability.-Equating (10) and (11) we have:

$$
\frac{\sqrt{2} a B_{a p p}}{(\cosh 2 m \alpha a-\cos 2 m \beta a)}=\frac{4 \pi \mu J}{m(\cosh 2 m \alpha a+\cos 2 m \beta a)^{\frac{1}{2}}}
$$

From this we obtain immediately an expression for the apparent permeability:

$$
\mu_{a p p}=\frac{\mu}{\sqrt{2} m \alpha} \frac{(\cosh 2 m \alpha a-\cos 2 m \beta a)^{\frac{1}{2}}}{(\cosh 2 m \alpha a+\cos 2 m \beta a)^{\frac{1}{2}}}
$$

This expression for the apparent permeability becomes identical with that given by J. J. Thomson when we assume $\tau=0$, that is to say $\alpha=\beta=1$.

It is necessary to know the expression for the apparent permeability, in order to determine the given or apparent magnetic induction, as a function of the available ampere-turns, in any given radio frequency apparatus.

III. We now proceed to determine the Foucault current and hysteresis losses as a function of the mean or apparent magnetic induction.

Foucault Current Losses.-In order to determine the Foucault current losses, it is necessary to determine in some manner the effective value in space of the current density $\boldsymbol{\delta}_{\max }$, that is to say, the value of:

$$
\frac{1}{\alpha} \int_{0}^{a} \delta_{\max }^{2} d x
$$

We have

$$
\begin{aligned}
\left(\check{\delta}_{\text {max }}^{2}\right)_{a v} & =\frac{A^{2}}{2 a} \int_{o}^{a}(\cosh 2 m \alpha x-\cos 2 m \beta x) d x \\
& =\frac{A^{2}}{4 m a}\left(\frac{\sinh 2 m \alpha a}{a}-\frac{\sin 2 m \beta a}{\beta}\right)
\end{aligned}
$$

Bearing in mind that the effective current density, as a func- 
tion of the time, is $\sqrt{2}$ times lower than the maximum density, the Foucault current losses per cubic centimeter will be:

$$
W_{F}=\frac{A^{2} \rho}{8 m a}\left(\frac{\sinh 2 m \alpha a}{a}-\frac{\sin 2 m \beta a}{\beta}\right)
$$

If we replace the constant $A$ by its value obtained from (10), we have,

$$
W_{F}=\frac{\omega^{2} a}{4 m g} \frac{\frac{\sinh 2 m \alpha a}{a}-\frac{\sin 2 m \beta a}{\beta}}{\cosh 2 m \alpha a-\cos 2 m \beta a} B_{a p p}^{2}
$$

The effect of hysteresis on Foucault current losses is readily seen. Leaving out hysteresis, the expression of these losses takes the simple form:

$$
W_{F}=\frac{\omega^{2} a}{4 m \varrho} \frac{\sinh 2 m a-\sin 2 m a}{\cosh 2 m a-\cos 2 m a} \boldsymbol{B}_{a p p}^{2} .
$$

Hysteresis Losses.- - To determine the hysteresis losses, we must bear in mind that the assumption of a constant phaselag $\tau$ of the magnetic induction $\boldsymbol{B}$ behind the magnetizing field $\boldsymbol{H}$ supposes the hysteresis losses to be proportional to the square of the maximum induction. It is therefore necessary to determine in some way the effective value of $\boldsymbol{B}_{\max }$ in space.

We have:

$$
\begin{aligned}
\left(\boldsymbol{B}_{\text {max }}^{2}\right)_{a x} & =\frac{A^{2} \varrho^{2} m^{2}}{\omega^{2} a} \int_{0}^{a}(\cosh 2 m \alpha x+\cos 2 m \beta x) d x \\
& =\frac{A^{2} \underline{\varphi}^{2} m^{2}}{2 \omega^{2} a}\left(\frac{\sinh 2 m \alpha a}{a}+\frac{\sin 2 m \beta a}{\beta}\right)
\end{aligned}
$$

We also know that the same hypothesis of constant phaseangle between the magnetic induction $\boldsymbol{B}$ and the magnetizing field $\boldsymbol{H}$ implies that, in the formula

$$
W=\eta \boldsymbol{B}_{m a x}^{2}
$$

which gives the hysteresis losses per cycle, the value of the coefficient $\eta$ is equal to $\frac{\sin \tau}{4 \mu}$. Under those conditions, the hysteresis losses per cubic centimeter are equal to:

$$
\begin{aligned}
W_{H} & =\frac{\sin \tau}{4 \mu} \frac{\omega}{2 \pi} \frac{A^{2} \varrho^{2} m}{2 \omega^{2} a}\left(\frac{\sinh 2 m \alpha a}{a}+\frac{\sin 2 m \beta a}{\beta}\right) \\
& =\frac{\sin \tau}{8} \frac{A^{2} \varrho}{m a}\left(\frac{\sinh 2 m \alpha a}{a}+\frac{\sin 2 m \beta a}{\beta}\right)
\end{aligned}
$$


If we replace the constant $A$ by its value from (10), we have:

$$
W_{H}=\frac{\omega^{2} a \sin \tau}{4 m \rho} \frac{\frac{\sinh 2 m \alpha a}{a}+\frac{\sin 2 m \beta a}{\beta}}{\cosh 2 m \alpha a-\cos 2 m \beta a} B_{a p p}^{2}
$$

Ratio of Losses.-The ratio of hysteresis losses to Foucault current losses can be readily determined. We find:

$$
\frac{W_{H}}{W_{F}}=\frac{\frac{\sinh 2 m \alpha a}{a}+\frac{\sin 2 m \beta a}{\beta}}{\frac{\sinh 2 m \alpha a}{a}-\frac{\sin 2 m \beta a}{\beta}} \sin \tau
$$

This ratio tends toward versin $\tau$ in proportion as the frequency increases.

Total Losses.-The expression for the total losses takes the form:

$$
W_{F}+W_{H}=\frac{\omega^{2} a}{4 m \varrho} \frac{\alpha \sinh 2 m \alpha a-\beta \sin \alpha m \beta a}{\cosh 2 m \alpha a-\cos 2 m \beta a} B_{a p p}^{2}
$$

If the calculation were not complicated by introducing the phase-angle $\tau$, and by seeking to determine the hysteresis losses according to the formula for losses per cycle (10), equation (14) could be simplified by replacing $\sin \tau$ by its value $4 \mu \eta$, and by making $\tau=0$, that is to say, by assuming $\alpha=\beta=1$ everywhere else in the equation; which would give the following value for $W_{H}$,

$$
W_{H}=\frac{\mu \eta \omega^{2} a}{\rho m} \frac{\sinh 2 m a+\sin 2 m a}{\cosh 2 m a-\cos 2 m a} \boldsymbol{B}_{a p p}^{2} .
$$

Taking into consideration the uncertainty which exists in regard to the exact value of hysteresis losses per cycle, and owing to the circumstances that all authors differ as to the value which should be given to the exponent of $\boldsymbol{B}$, to which the losses are proportional, the simplified expression for $W_{H}$, given in equation $\left(14^{\prime}\right)$, may often be utilized.

Under those conditions, the total losses derived from $\left(13^{\prime}\right)$, and $\left(14^{\prime}\right)$, will be as follows:

$$
W_{F}+W_{H}=\frac{\omega^{2} a(1+4 \eta \mu) \sinh 2 m a}{4 m g \cosh 2 m a-\cos 2 m a} B_{a p p}^{2} .
$$

Minimum Losses.-It is possible to determine the losses per cubic centimeter in a total volume containing the iron sheets and the insulation between them.

Let $\varepsilon$ be the thickness of the insulation between the sheets, 
and $\boldsymbol{B}_{a p p}^{\prime}$ the mean magnetic induction in the total section composed of the sheets and of the insulation between them. The apparent induction in the sheet itself will be:

$$
\frac{2 a+\varepsilon}{2 a} B_{a p p}^{\prime}
$$

On the other hand, the space occupied by the iron will be reduced in the proportion $\frac{2 a}{2 a+\varepsilon}$. Finally, the losses per cubic centimeter of total space will be, from (16), as follows:

$$
W_{F}+W_{H}=\frac{\omega^{2}(2 a+\varepsilon) \alpha \sinh 2 m \alpha a-\beta \sin 2 m \beta a}{8 m ! \cosh 2 m a a-\cos 2 m \beta a} \boldsymbol{B}_{a p p}^{\prime 2}
$$

The thickness of sheets $2 a_{\text {opt }}$ which will give the minimum of loss in a given volume for a given magnetic induction $B_{a p p}^{\prime}$ will be that which will always give the minimum value to the preceding expression. This minimum value is obtained-by taking the derivative of that expression with respect to " $a$." In other words, it will be that thickness which represents the solution of the following transcendental equation:

$2 m(2 a+\varepsilon) \frac{1-\cosh 2 m \alpha a \cos 2 m \beta a}{\cosh 2 m a a-\cos 2 m \beta a}+\alpha \sinh 2 m \alpha a$

$$
-\beta \sin 2 m \beta a=0
$$

As a numerical illustration, let us take:

$$
\begin{aligned}
\mu & =2000 \\
\rho & =4(10)^{4} \\
\omega & =2 \pi \times 30,000 \\
\varepsilon & =0.003
\end{aligned}
$$

From this we have:

$$
m=\sqrt{\frac{2 \pi \mu \omega}{\rho}}=243.5
$$

We then find for $2 a_{\text {opt }}$ the following values:

$$
\begin{array}{ll}
\text { With } \sin \tau=0.2 & 2 a_{\text {opt }}=0.027 \mathrm{~mm} .(0.001 \mathrm{inch}) \\
\text { With } \sin \tau=0.3 & 2 a_{\text {opt }}=0.0315 \mathrm{~mm} .(0.0012 \mathrm{inch}) \\
\text { With } \sin \tau=0.5 & 2 a_{\text {opt }}=0.0375 \mathrm{~mm} .(0.0014 \mathrm{inch})
\end{array}
$$

\section{Phase-Angle Between EMf. and Current in an Induc-} tance Having a Closed Magnetic Circuit

The losses have been evaluated directly without seeking to determine, as is usually done, the phase-angle $\phi$ between the emf. and the current. When the losses are known this 
phase-angle can be determined quite easily by an inverse process.

The maximum emf. " $V$," induced per turn per square centimeter will be $\omega \boldsymbol{B}_{a p p}$, and the power consumed per cubic centimeter will, therefore, be:

$$
\frac{V J}{2} \cos \phi=\frac{\omega B_{a p p} J}{2} \cos \phi
$$

On the other hand, we know this power from equation (16) and we also know the value of $\boldsymbol{B}_{a p p}$, as a function of $J$, from that of the apparent permeability. Equating these two expressions for losses, the value of $\cos \phi$ may be readily obtained. We will have:

$$
\cos \phi=\frac{1}{\sqrt{2}} \frac{\alpha \sinh 2 m a a-\beta \sin 2 m \beta a}{\left(\cosh ^{2} 2 m \alpha a-\cos ^{2} 2 m \beta a^{\frac{1}{2}}\right.}
$$

When $m$ tends toward infinity $\cos \phi$ tends toward $\frac{a}{\sqrt{2}}$; or $\sqrt{\frac{1+\sin \tau}{2}}$; that is to say, $\phi$ tends toward the angle $\frac{\pi}{4}-\frac{\tau}{2}$, as Mr. Bethenod has shown. We have in fact:

$$
\sqrt{\frac{1+\sin \tau}{2}}=\cos \left(\frac{\pi}{4}-\frac{\tau}{2}\right)
$$

\section{Phase-Angle Between EMF. and Current in a Coil with Open Magnetic Circuit}

In a coil having an open magnetic circuit, which includes an air-gap that multiplies the apparent reluctance of the magnetic circuit by $k$, the magnetic induction for the same ampereturns, $J$, will be divided by $k$. Consequently, the losses are divided by $k^{2}$, while the emf. induced at the terminals is divided by $k$. Under those conditions it will be found that the phaseangle $\phi^{\prime}$ becomes such that:

$$
\cos \phi^{\prime}=\frac{\cos \phi}{k}
$$

in which $\cos \phi$ retains the value indicated in the preceding paragraph.

In proportion as the air-gap is increased, the emf. and the current tend more and more to assume the quarter-phase relation. As a rule, it is $\tan \phi^{\prime}$ which should be given as high a value as possible.

The author will return later to the important question of the construction of inductance coils with low losses (with or without iron) for a given frequency. 
SUMMARY: In this article there is determined the power dissipated separately by Foucault currents and by hysteresis, in a sheet of iron, on the assumption that there exists a constant angle of lag between the magnetic induction in the sheet and the magnetizing field producing it. There is deduced the thickness which should be given to the iron sheets of apparatus supplied with radio frequency current, in order that the total power expended shall be a minimum. A calculation is made of the angle of lag between the voltage and the current in the circuit of an inductance coil. 